\section{Ernst Schneider}

V itis vinifera, die Weinrebe liefert uns den allseits bekannten Rotwein. Weit weniger geläufig ist dagegen die Verwendung von rotem Weinlaub. Dabei haben die vielfältigen Anwendungen von Weinblättern in der Medizin eine lange Geschichte, ohne dass rotes Weinlaub explizit erwähnt wird. Dieses taucht erst in unserer Zeit in der medizinischen Literatur auf. Seit einigen Jahrzehnten gehört ein Extrakt aus roten Weinblättern mit der Indikation Vorbeugung und Behandlung chronischer Venenschwäche zu den bekannten Phytopharmaka am deutschen Markt. In Frankreich sind rote Weinblätter unter der Bezeichnung «Vigne rouge» sehr populär, wie die Vielzahl von Produkten belegt.

Begeben wir uns auf die Reise durch die Geschichte und suchen nach historischen Belegen für die Anwendung von Weinlaub, insbesondere von rot gefärbten Blättern und wie weit ihre heutige Verwendung für die Indikation Venenerkrankungen zurückzuverfolgen ist.

\section{Paläobotanische Belege}

Der wohl älteste Nachweis von Weinblättern in Verbindung mit menschlichen Siedlungen stammt aus Bilzingsleben, einem Fundort von Homo erectus aus der Zeit vor ca. 350’000 Jahren. Im Travertin wurden die Funde zusammen mit Resten der damals vorkommenden mediterranen Vegetation konserviert. Über die Verwendung kann allerdings nur spekuliert werden, da die Fundumstände keine weiteren Informationen dazu liefern [43]. Während der atlantischen und subborealen Periode (7500-2500 Jahre zurück) sind archäologische Funde von Traubenkernen der Wildrebe $V$. vinifera subsp. sylvestris für weite Teile Europas belegt [65]. Der Beginn der Geschichte der Weinkultur ist noch nicht genau ge-

\title{
Rotes Weinlaub - Geschichte der Verwendung in der Medizin
}

Weingenuss ist allgemein bekannt, ganz im Gegensatz zur Verwendung von Weinblättern. Dabei werden Extrakte aus rotem Weinlaub heute als Phytopharmaka bei chronisch-venöser Insuffizienz häufig eingesetzt. Die medizinische Anwendung von Weinlaub ist seit der Antike belegt. Durch genaue Rückverfolgung der medizinischen Literatur aus Frankreich war es möglich, erste Hinweise im Zusammenhang mit dem Auftreten von rot beblätterten Weinstöcken im 17. Jahrhundert zu finden.

klärt. Sicherlich wurden die Trauben zunächst als Obst geschätzt. Aufgrund der natürlich vorhandenen Weinhefe gären Trauben von selbst, weshalb die Weinbereitung von der Natur schon vorgegeben ist. Erste Spuren für die Kultivierung von Reben fanden sich aus der Zeit um 5000 v.Chr. im Transkaukasus und an der Schwarzmeerküste. Um 3000 v.Chr. ist dann Weinbau zunächst in Kleinasien, etwas später dann auch in Ägypten fassbar. Von dort verbreitete sich diese Kulturpflanze über den Mittelmeerraum und hat um 300 n.Chr. Zentraleuropa erreicht [65].

Im Alten Testament heisst es: «Noah aber, der Landmann, war der erste, der Weinreben pflanzte» (1. Mose 9,20). Die Bibel beschreibt also, dass Wein schon zu Beginn der Geschichte der Menschheit angebaut wurde. Dieses Motiv ist beliebter Gegenstand von $\mathrm{Ab}$ bildungen in Bilderbibeln, wobei auch die Wirkung des zu reichlichen Weingenusses dargestellt wird [4].

Untrennbar mit der Verbreitung der Rebkultur im östlichen Mittelmeergebiet verbunden ist die Ausbreitung des Dionysos-Kultes. Seine Blüte erlebte er, als zwischen 2200 und 1400 v.Chr. der Weinbau Griechenland und den Balkan erreichte. Es handelt sich hierbei um einen ausgelassen gefeierten Kult der jährlichen Erneuerung der Natur. Bildliche Darstellung dieser Erneuerung ist der Weinstock im Bogen.

Im alten Ägypten war Wein sehr beliebt und der Weinbau florierte seit 1400 v.Chr., wie farbige Wandmalereien in den Gräbern zeigen. Im Grab des Nakht wird ein Weinstock in der eben angesprochenen Form einer bogenför- migen Pergola dargestellt. Interessant ist die Nennung der innerlichen medizinischen Anwendung von Traubenkernen bei «Hitze im After» im Papyrus Bt23, also bei hämorrhoidalen Beschwerden [20], die durch deren Gehalt an gefässwirksamen Procyanidinen erklärbar wird [10].

Im ersten Jahrtausend v.Chr. verbreitete sich der Weinbau mit den Seefahrern der Phönizier und Griechen nach Nordafrika, Süditalien, Frankreich und Spanien [11]. Die Römer brachten den Weinbau an den Rhein und um 300 n.Chr. war die Weinherstellung dann in ganz Europa gebräuchlich. In Nordamerika begann man damit um 1850 .

\section{Medizinische Anwendung in der Antike}

Dioskurides Pedanios aus Anazarbos berichtet im fünften Buch seines zwischen 60 und 78 n.Chr. datierten Werkes «De Materia medica» ausführlich über Wein, wobei der Weinstock mit dem griechischen Wort ampelos für Weinrebe belegt wird. Als Umschlag sollen die zerkleinerten Blätter und Ranken Kopfschmerzen, mit Graupen vermengt eingenommen auch Entzündung und Brand des Magens lindern. Allein aufgelegt, wirken sie kühlend und adstringierend. Der aus ihnen gepresste Saft hilft innerlich bei Dysenterie, Blutauswurf, Magenschmerzen und falschem Appetit schwangerer Frauen [5]. Die Wertschätzung von Weinlaub bei den Griechen erkennt man auch daran, dass auf antiken griechischen Münzen Weinblätter häufig abgebildet sind [3]. 
Claudius Galenus (129-199 n.Chr.), der griechische Arzt in römischen Diensten, beschreibt mehrmals die Verwendung von Weinblättern in seinen Rezepturen, weshalb davon auszugehen ist, dass deren medizinische Verwendung damals üblich und verbreitet war. Nach seinen Empfehlungen helfen geriebene Weinblätter, in Honigwein gekocht, äusserlich gegen Kopfschmerz, der durch Hitze hervorgerufen wird. Als ein kühlendes Vorbeugemittel in heissen Gegenden werden Weinblätter mit Ranken (pampinorum vitis) empfohlen. Auf geschwollene, entzündete Geschlechtsteile werden junge Weinblätter als Umschlag gelegt und zerriebene grüne Weinblätter sorgen für guten Magen, wenn sich zu viel Speichel angesammelt hat [34].

Plinius Secundus (23-79 n.Chr.) hat man wegen seiner 37 Bücher umfassenden Naturgeschichte auch den «Brockhaus des klassischen Altertums» genannt. Im 23. Buch über die pharmakologischen Wirkungen der Kulturpflanzen zählt er die Weinblätter neben anderen Teilen des Weinstrauchs auf. Die Blätter wirken danach bei Kopfweh, gegen Entzündungen, bei brennendem Schmerz im Magen, bei Gelenkerkrankungen, gegen Blutfluss aus Wunden und heilungsfördernd bei entzündeten Wunden [33].

\section{Mittelalter / frühe Neuzeit}

In der mittelalterlichen Literatur wird der Weinstock als Metapher für Christus mehr der christlichen Heilslehre zugeordnet und nur selten ist auch eine medizinische Indikation zu erkennen. Bei Hildegard von Bingen wird der Weinstock mit der Bezeichnung «Vitis» mehrmals aufgeführt, allerdings sind keine Hinweise auf die Verwendung von Blättern $\mathrm{zu}$ finden [53,56]. Im «Liber floridus», von dem Kanoniker Lambertus von Saint-Omer um 1120 in Flandern verfasst, wird der Weinstock im religiösen Zusammenhang dargestellt und auf die achte Seligpreisung der Bergpredigt verwiesen (Abb. 1). Verfolgung des Glaubens wegen gilt demnach als höchste Vollendung, weil sie wie der Weinstock Früchte trägt [35].

Albertus Magnus (1198-1280), des-

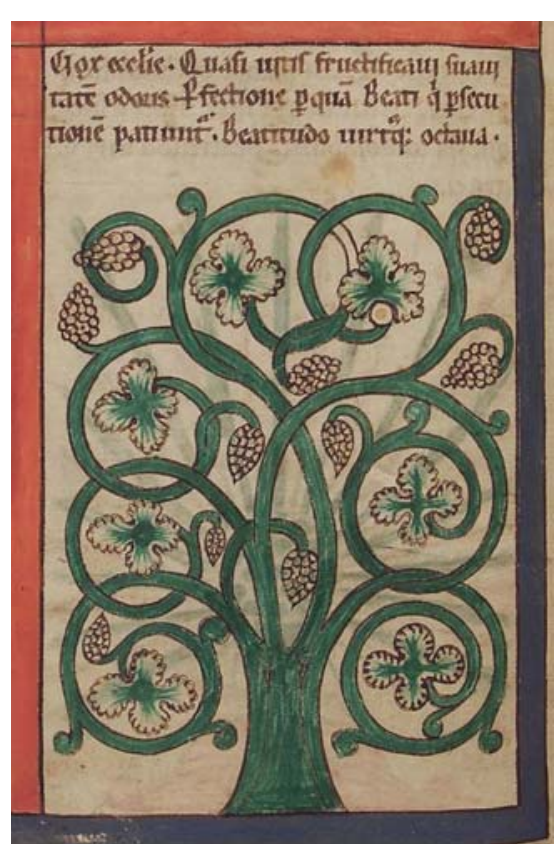

Abb. 1. Abbildung eines Weinstocks im Liber floridus des Lambertus von St-Omer. Entstehungszeit: ca. 1120. Blatt 158 aus Cod. Guelf. 1 Gud. lat., Herzog August Bibliothek, Wolfenbüttel.

sen naturwissenschaftliche Kenntnisse aussergewöhnlich waren, hat das Weinlaub für die damalige Zeit sehr ausführlich und in seiner heilkundlichen Wirkung beschrieben [27]. Er charakterisiert es als kalt und trocken sowie zusammenziehend und bezieht sich dabei auf Dioskurides. Den Saft der Blätter empfiehlt er bei Dysenterie sowie die Asche als Hämorrhoidenmittel und bei Analgeschwüren. Der sogenannte deutsche Bartholomäus, eine mittelalterliche Handschrift, führt Weinblätter als Mittel gegen Schlaflosigkeit an [15].

Im Mittelalter waren bei Laien die Tacuinum-sanitatis-Handschriften, auch «Schachtafeln der Gesundheit» genannt, sehr beliebt. Diese Gesundheitsbücher in tabellarischer Übersicht gehen auf das arabische Werk «Taqwin As-Sihha» des Arztes Ibn Bôtlan, zurück, der in der Mitte des 11. Jh. in Bagdad lebte. Wein und Weintrauben werden in ihrer Anwendung und Eigenschaft beschrieben, nicht jedoch Weinblätter [44]. Der Weinbau ist darin in farbenprächtigen abendländischen Miniaturen illustriert.

Weitere Angaben zur Verwendung des Weinstocks liefert der Regensburger Domherr und Ratsherr Conrad von Megenberg (1309-1374) in seinem «Buch über die Natur», wo der Weinstock auf dem Frontispiz «Von den Bäumen» abgebildet wird [45]. In Kapitel 54 «Von den Weinreben» werden die grünen Triebe mit Blättern bei Augenleiden und tränenden Augen empfohlen.

\section{Zeit der gedruckten Kräuterbücher}

In einem der ersten gedruckten Kräuterbücher, dem «Gart der Gesundheit» (1485) des Frankfurter Stadtarztes Johann Wonnecke von Kaub (Johann von Cube) wird berichtet, dass Serapion, ein arabischer Arzt im 11. Jh., den Saft von Weinblättern bei Darmgeschwür und Blutspeien verwendet [63]. Der Autor hat entgegen bisheriger Lehrmeinung nicht nur aus deutschen, sondern auch aus lateinischen Quellen geschöpft - das Buch ist damit eine Art Quintessenz des gesamten mittelalterlichen botanischen Wissens [24]. Ein Nachdruck dieses Werkes erschien in Venedig 1511 unter dem Titel «Ortus sanitatis«, in dem die Blätter bei Intestinalulcera, zur Blutstillung sowie bei Appetitlosigkeit von Schwangeren verwendet werden [50].

In den damals sehr beliebten bebilderten Kräuterbüchern der «Väter der Botanik» durfte der Weinstock natürlich nicht fehlen [14]. Der Botaniker, Arzt und Theologe Hieronymus Bock (1498-1554) schreibt 1546 in seinem Buch: «Das Rebenlaub/ sampt den hefftlein/ zerstossen/ und übergelegt/ stillet das grausam Hauptweh/ löschet alle hitz des haupts/ der Augen/ und magen». 1577 schreibt er: «Die hafftlein oder gabelein an den Reben/zerstossen/ und den safft eingetrunken/ stillet den rothen bauchfluss. Gemelte würckung hat der safft von laub/ und das gebranndt wasser von ihnen beiden» [9].

Rembert Dodoens (Dodonaeus, 15171585) hat sein «Cruydt-boeck» erstmals 1554 in Flämisch in Antwerpen verlegt. In diesem Kräuterbuch, das auf den italienischen Arzt Pietro Andrea Mattioli (Matthiolus, 1501-1577) zurückgeht, steht unter Vitis, dass die Blätter von trockener Natur sind und adstringierende Wirkung zeigen [13].

In der deutschen Ausgabe des «Kreuterbuch» von Adam Lonitzer (Lonicerus, 1527-1586) wird zu Weinblättern vermerkt: «Das Rebenblat heisst Pampinus. Natur oder Complexion. Der 
Saft von Blättern getruncken/ ist gut wider die rohte Ruhr/ wider das Blutspeyen/ Magens wehthum/ und das böss gelüsten der Weiber/ welches man Citam und Picam nennet. Die Blätter und Gäblin gestossen und aufgestrichen/ sänftigen den Schmwertzen dess Haupts/ und des Magens Entzündung. Dergleichen thun die Blätter/ für sich selbst aufgelegt/ dann sie kühlen und ziehen zusammen» [41].

In dem prachtvoll bebilderten Pflanzenbuch «De historia stirpium» (in der deutschen Ausgabe «New Kreüterbuch», 1543) von Leonhart Fuchs (15011566) heisst es: «Die Rebbletter und zincken zerstossen/ unnd übergelegt/ lindern den wee tagen des haubts. Mit gersten maltz vermengt/ miltern sie die entzündung und die überige hitz des magens. Sölche würckung haben auch die bletter allein unnd für sich selbst übergelegt» [17] (Abb. 2).

Jakob Horst schreibt in seinem 1630 in Marburg erschienenen «Opusculum de vite vinifera ejusque partibus», dass frische Weinblätter sich als Pflaster eignen und Ulzerationen am Kopf und anderen Muskelpartien heilen. Abgefallene Blätter auf trockene Krätze aufgelegt, vertreiben die Milben und erweichen den Schorf. Reblaub, auf die Stirn gelegt, soll bei Kopfweh helfen [26].

Tabernaemontanus zitiert 1731 die Angaben von Dodoens und zusätzlich: «Dessgleichen werden sie auch nutzlich gebraucht/ wider die Bauchfluss und rothe Ruhr/ wider das Würgen des Magens. Sie werden auch gebraucht wider die Hitz und Entzündung des Magens/ wider den Unwillen/ und unnatürliche Lüsten der schwangeren Weiber: Entweder äusserlich gebraucht/ oder aber das Wasser oder den Saft darvon innerlich eingenommen» [59].

Alle bisher angeführten Werke zitieren mehr oder weniger die bei Dioskurides schon aufgelisteten Indikationen, wobei auch immer wieder die Wirkung bei gefässbedingten Beschwerden hervorgehoben wird (Tab. 1).

\section{Geschichte der botanischen Beschreibung}

Die Gattung Vitis gehört zur Pflanzenfamilie der Vitaceae, Weinrebenge-

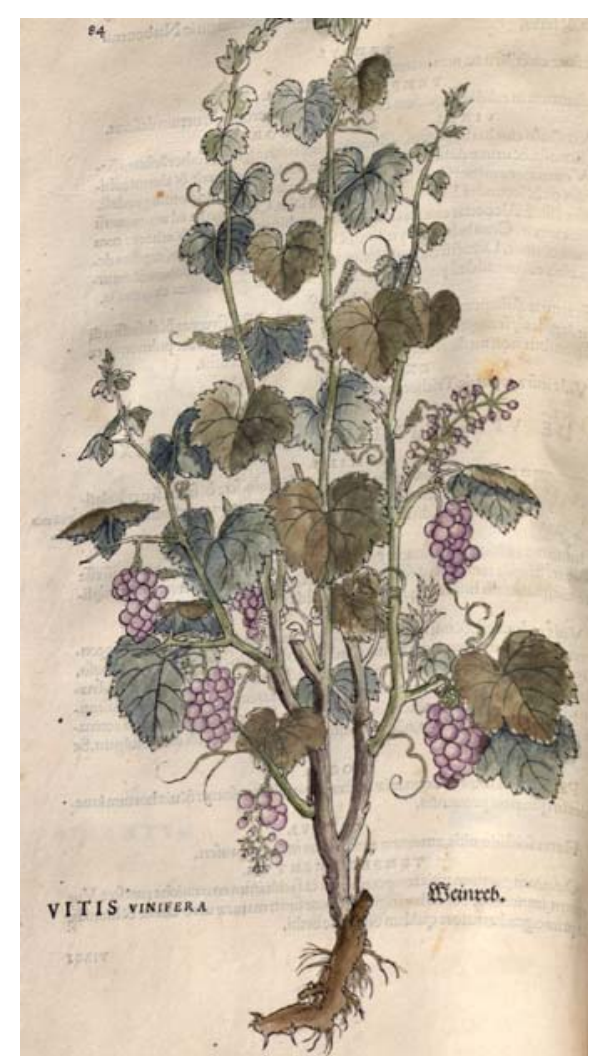

Abb. 2. Abbildung des Weinstocks im Kräuterbuch De historia stirpium von Leonhart Fuchs, Basel 1542 (Universitätsbibliothek Regensburg). Die Kolorierung weist für die Blattoberseite einen rötlichen Ton auf.

wächse, von der zwei Unterarten unterschieden werden: die ursprüngliche Wildform Vitis vinifera subsp. sylvestris (Gmel.) Beger und Vitis vinifera subsp. vinifera, die Kultur- oder Edelrebe. Die Wildform hat typisch geformte, breit birnenförmige, hartschalige Samen im Gegensatz zur Kulturrebe mit schlanken, meist länglich birnen- oder eiförmigen Kernen. Dieser Unterschied dient den Archäologen als Beleg für das Auftreten der Kulturrebe und ihre Verbreitung.

Die Namensgebung Vitis vinifera L. geht auf Carl von Linné zurück [40]; er bezieht sich dabei auf Angaben von Caspar Bauhin in dessen «Pinax Theatri botanici» von 1671, wo verschiedene Arten, Sorten und deren Anbaugebiete genannt werden [2]. Auch bei Tournefort werden bereits 1719 viele Vitis-Arten angegeben und erstmals die Pflanzenorgane detailliert abgebildet [60]. Diese Detailtreue wurde erst über 100 Jahre später in der Zeit der pharmakognostischen Atlanten übertroffen (Beispiel bei Otto C. Berg [6]).
Neuere Zeit -

\section{Weinlaub in der Pharmazie}

In einem als «Keller-Meister» bezeichneten anonymen Werk von 1705 steht [29]: «Aus den zarten Blättern/ im Mayen/wird ein Wasser destilliret/ welches den Schwangeren/ die nach einer Speise gelüstet/ und solche nicht haben können/ nutzlich gegeben wird/ dass der Frucht nicht schaden bringe. Das Pulver/ von den Reben/ in die Nasen gethan/ oder getruncken/ solle trefflich seyn/ wider das Nasen-Bluten».

Der «Allgemeine Artzney-Schatz» des Johann Schröder von 1748 berichtet über den Weinstock, dass «man in den Apothecken die Blätter hat», diese also damals offizinell waren. Weiter wird ausgeführt: «Die Blätter kühlen und adstingiren sehr, werden gebraucht in der rothen Ruhr, dem Erbrechen, Blutausspeyen und andern Blutflüssen. Eusserlich kühlen und lindern sie die Haupt-Schmerzen und Hitze, bringen den Schlaff, wenn man die Füsse mit wäschet, oder selbe dem Haupt appliciert. Die Blätter haben einen lieblichen sauren Saft in sich, daher sie wider das Hauptwehe und Hitze grossen Ruhm verdienen. Man bindet sie auf die Fusssohlen den Schlaff zu befördern; auch kochet man sie in den FussBädern. Sie haben zugleich in etwas eine adstringirende Krafft, weswegen man sie in den Diarrhoeen und Dysenterien nützlich gebrauchet; so befördern sie auch den verlohrnen Appetit» [57].

Ob Weinblätter in Apotheken wirklich üblich waren, bleibt fraglich, da sie weder in der Lüneburger Warenlagerliste von 1475, noch in der Württembergischen Taxe von 1626 aufgeführt sind. Im Köhler-Atlas (1887) wird angemerkt, dass alle Teile des Weinstocks schon seit frühster Zeit arzneilich verwendet wurden [51], dass die frischen Blätter offizinell waren und zum Teil noch sind. Allerdings werden diese im weiteren Verlauf des Kapitels weder bei Anwendung, noch bei den Arzneibuchmonographien erwähnt. Erst in jüngster Zeit wurden sie nachweislich offizinell. In der ersten Ausgabe des Homöopathischen Arzneibuchs von 1934 werden frische Weinblätter aufgeführt [58] und ab 1996 ist rotes Weinlaub als Monographie «Vigne rouge» 


\section{Fortbildung}

Tab. 1. Zeitliche Folge gefässbezogener Indikationen für Weinlaub

\begin{tabular}{|c|c|c|}
\hline Zeit & Autor & Indikation \\
\hline \multicolumn{3}{|l|}{ Altes Ägypten } \\
\hline 1400 v.Chr. & Papyrus Bt23 & Hitze im After \\
\hline \multicolumn{3}{|l|}{ Antike } \\
\hline 60-78 n.Chr. & Dioskurides & Entzündung und Brand des Magens, Blutauswurf \\
\hline 23-79 n.Chr & Plinius Secundus & Entzündungen, Blutfluss aus Wunden, entzündete Wunden \\
\hline 129-199 n.Chr. & Claudius Galenus & entzündete Geschlechtsteile \\
\hline \multicolumn{3}{|l|}{ Mittelalter } \\
\hline $1198-1280$ & Albertus Magnus & als Hämorrhoidenmittel, bei Analgeschwüren \\
\hline $1309-1374$ & Conrad von Megenberg & Augenleiden, tränende Augen \\
\hline 1485 & Johann Wonnecke von Kaub & Darmgeschwür, Blutspeien \\
\hline \multicolumn{3}{|l|}{ Neuzeit } \\
\hline 1511 & Ortus sanitatis & bei Intestinalulcera, zur Blutstillung \\
\hline 1546 & Hieronymus Bock & $\begin{array}{l}\text { stillet den rothen bauchfluß } \\
\text { löschet alle hitz des haupts / der Augen / und magen }\end{array}$ \\
\hline $1527-1586$ & Adamus Lonicerus & wider die rohte Ruhr / wider das Blutspeyen \\
\hline $1501-1566$ & Leonhart Fuchs & miltern sie die entzündung und die überige hitz des magens \\
\hline 1630 & Jakob Horst & $\begin{array}{l}\text { Wundpflaster } \\
\text { sanare ulcera capitis \& aliarum musculosarum partium }\end{array}$ \\
\hline 1731 & Tabernaemontanus & $\begin{array}{l}\text { wider die Bauchfluß und rothe Ruhr, } \\
\text { Entzündung des Magens }\end{array}$ \\
\hline 1705 & Keller-Meister & wider das Nasenbluten \\
\hline 1748 & Johann Schröder & in der rothen Ruhr, Blutausspeyen und andern Blutflüssen, Nasenbluten \\
\hline $1804(1730)$ & J.B. Chomel & bei Regelbeschwerden \\
\hline Ende 19. Jh. & M. Camel & normalisiert zu starke und lang dauernde Monatsblutungen (rotes Weinlaub) \\
\hline \multicolumn{3}{|l|}{ 20. Jahrhundert } \\
\hline 1942 & Leclerc $[36]$ & $\begin{array}{l}\text { schmerzhafte Venenentzündungen Hämorrhoiden, Gynäkologikum } \\
\text { (rotes Weinlaub) }\end{array}$ \\
\hline 1943 & Godlewsi \& Blanque [21] & Venenmittel, Gynäkologikum (rotes Weinlaub) \\
\hline 1948 & Fournier [16] & Bluterguss, Krampfadern, Nasenbluten, Uterusblutungen (rotes Weinlaub) \\
\hline 1953 & Zicavo [64] & $\begin{array}{l}\text { Krampfadern, lokale Durchblutungsstörungen, Vitamin-P-Wirkung } \\
\text { (rotes Weinlaub) }\end{array}$ \\
\hline 1957 & Bertone [8] & Gefäßerkrankungen in der Gynäkologie, Kapillarbrüchigkeit (rotes Weinlaub) \\
\hline 1969 & Antistax ${ }^{\circledR}$ registriert & $\begin{array}{l}\text { Behandlung chronisch-venöser Insuffizienz (CVI), Krampfadern, } \\
\text { Beinödeme, schwere Beine (rotes Weinlaub) }\end{array}$ \\
\hline
\end{tabular}

im französischen Arzneibuch enthalten [52]. In den neuesten deutschen Lieferlisten für Apotheken (Galke-Liste 2007) sind Weinblätter aufgeführt, während man sie in solchen von 1994 noch vergeblich sucht (Caelo-Liste 1994).

In Deutschland wird frisches Weinlaub ohne Sortenangabe in der Homöopathie unter der Bezeichnung «Vitis vinifera-Wein» verwendet [25,58]. Die Monographie der Kommission D stuft Weinblätter als homöopathisch nicht wirksam ein, was auf eine geringe Bedeutung in der Homöopathie hindeutet
[31]. Neuere Publikationen suchen diese Lücke durch Prüfung des homöopathischen Arzneimittelbilds für das Wirkprofil «Geist/Gemüt» zu schliessen [32].

\section{Die Färberrebe, Quelle für rotes Weinlaub}

Die als Färber verwendeten Rebsorten für Deckweine zeichnen sich durch eine Rotfärbung sowohl der Blätter als auch des Safts der Trauben aus - im Unterschied zu üblichen Rotweintrau- ben, die hellen Saft aufweisen. Diese Deckweine werden zur Farbvertiefung bei farbschwachen Rotweinen verwendet. Die Urtraube der Färbertrauben ist die französische Teinturier $d u$ Cher (Abb. 3). Sie wurde wahrscheinlich aus einer Wildrebe selektioniert und schon im 17. Jahrhundert in der Gegend von Orléans als Farbgeber bei Weinverschnitten verwendet [19]. Daneben wird noch eine weitere, ebenfalls sehr alte Sorte beschrieben: Teinturier male. Beide Färbertrauben werden in Rebbüchern üblicherweise zusammen ab- 


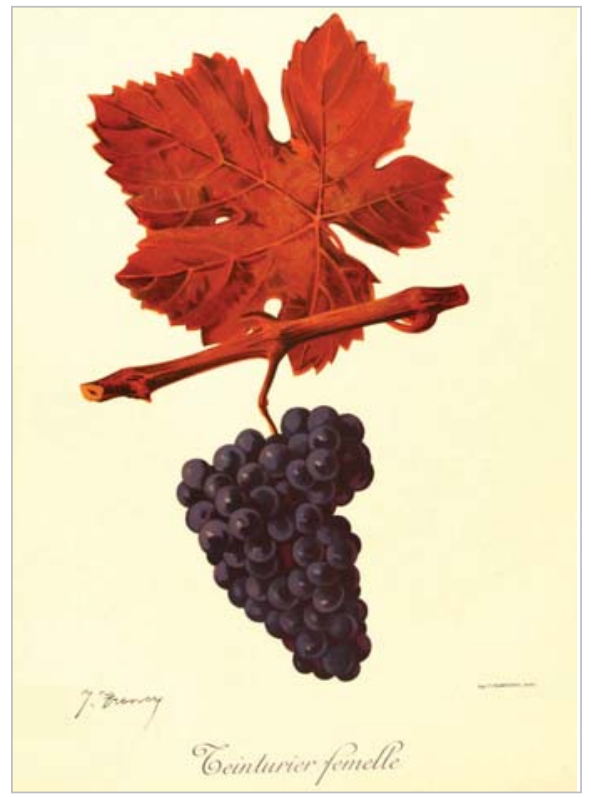

Abb. 3. Die rote Rebsorte Teinturier du Cher (Syn.: Teinturier femelle) gilt als Ursprung aller neugezüchteten Färbertrauben. Abbildung aus der Ampélographie von Viala (1905-1910) [61].

gehandelt und häufig verwechselt, unterscheiden sich aber in Form und Farbe der Blätter, sowie in der Farbtiefe des Saftes [18,61].

Aus diesen beiden gingen die heutigen französischen Rebsorten für Deckweine Gamay teinturier, Petit Bouschet und Alicante Bouschet (Abb. 4) hervor. Färberreben und ihre Neuzüchtungen kamen erst so richtig in Gebrauch, als aufgrund der Reblauskatastrophe im 19. Jahrhundert nur farbschwache Rotweinreben überlebten, deren Wein einer Farbvertiefung bedurfte [18].

\section{Geschichte der Anwendung von rotem Weinlaub}

Angesichts der seit der Antike belegten Verwendung von Weinlaub bei Anwendungen, die mit Blutungen, Wunden, Entzündungen, Hämorrhoiden und dem Gefässsystem zu tun haben, könnte man im Sinne der Signaturenlehre an die Analogie «rotes Blut - rotes Weinlaub» denken [47]. Aber suchen wir weiter nach Belegen für die medizinische Verwendung von rotem Weinlaub.

Der schon zitierte Kräuterbuchautor Caspar Bauhin erwähnt 1671 zwar in seinem «Pinax», dass manche Weinstöcke rote Blätter tragen, allerdings ohne Angaben zu einer medizinischen

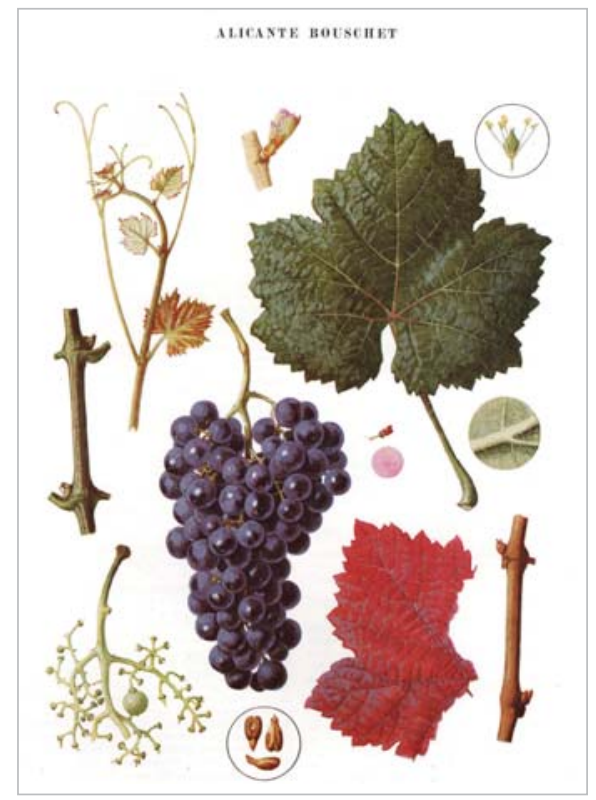

Abb. 4. Farbtafel zur Ampelographie von Alicante Bouschet (aus [49], Forschungsanstalt Geisenheim Hauptbibliothek). Deutlich ist zu erkennen, dass bei dieser Sorte nur das Herbstlaub rot gefärbt ist.

Indikation zu machen. Etwa um dieselbe Zeit empfiehlt Fumanelli aus Verona gegen Dysenterie und Erbrechen Weinblätter, die zu sammeln sind, wenn sie sich rot verfärben [36]. M. Camel, ein praktischer Arzt aus dem französischen Chaumont-en-Vexin, wendet gegen Ende des 19. Jahrhunderts rote Weinblätter an, um bei Frauen zu starke und lang dauernde Monatsblutungen zu normalisieren [36].

In einer Untersuchung zur Ethnobotanik von Frankreich wird angegeben, dass in der Provence (Hautes-Alpes) und im Süden Korsikas Weinblätter traditionell bei Veneninsuffizienz, Krampfadern und Hämorrhoiden eingesetzt werden [22] und in der Volksmedizin dieser Region sind rote Weinblätter in Blutreinigungsmitteln als «vasoconstructeur» enthalten [39].

Daher erstaunt es nicht, dass rotes Weinlaub auch erstmals in Frankreich von Ärzten in der Therapie verwendet wird. Henri Leclerc, der als einer der Begründer der modernen Phytotherapie in Frankreich gilt, beschreibt 1942 einen Tee aus roten Weinblättern als klassisches Heilmittel bei Beschwerden in der Menopause [36]. In seinem kurz darauf erschienenen Lehrbuch «Précis de Phytothérapie» verweist er auf Publikationen über Therapieerfolge mit einem alkoholischen Extrakt aus rotem
Weinlaub bei schmerzhaften Venenentzündungen und Hämorrhoiden [37].

\section{Alternativen für Importdrogen gesucht}

Dies geht zurück auf eine Veröffentlichung von Godlewsi und Blanque 1943 mit Fallbeschreibungen und Therapieerfahrungen mit rotem Weinlaub bei schmerzhaften Regelbeschwerden, Krampfadern und Hämorrhoiden [21]. In dieser Arbeit wird auch die Frage geklärt, wann und warum erstmals rote Weinblätter für diese Indikation eingesetzt wurden: Bedingt durch den 2. Weltkrieg waren die französischen Ärzte von den gewohnten pflanzlichen Gynäkologika und Venenmitteln, wie Hydrastis canadensis, Hamamelis virginiana, Viburnum prunifolium und Gossypium herbaceum, abgeschnitten, da sie meist aus Amerika importiert wurden. Daher suchte man in der französischen Flora nach Ersatzdrogen [21]. Aufgrund eines Hinweises in einem Buch von J.B. Chomel aus dem Jahr 1804 [12] auf die Verwendung eines Blätterpulvers von Muskatellertrauben bei Monatsbeschwerden, stiessen die Autoren auf die volkstümliche Verwendung von Weinlaub. Zu ihrem Erstaunen ergab eine Nachfrage bei Kräuterhändlern, dass diese im Herbst schon immer grosse Mengen roter Blätter sammelten, die in der Volksmedizin als Blutreinigungsmittel und Adstringens benutzt wurden [21]. Dies ist auch der Grund, warum man stolz darauf verweist, die Anwendung von rotem Weinlaub sei eine französische Erfindung [22].

Bleibt noch zu klären, wie es zu dem Indikationswandel von den Frauenbeschwerden zur Venenwirksamkeit kam. Hypermenorrhoe interpretierte man damals als eine Erscheinung, die auf venöse Stauungen durch Blutandrang zurückzuführen ist. Aufgrund des Gehaltes an Gerbstoffen, die bei der Extraktion noch angereichert werden, wirken rote Weinblätter tonisierend auf die Venen und sind daher bei derartigen Problemen des venösen Blutflusses angezeigt [21].

Im Kapitel «Vigne-Vitis» im Buch der Arzneipflanzen Frankreichs von P. Fournier [16] werden die roten Blätter von Weinstöcken der «Teinturier» ge- 
nannten Sorten (s.o.) wegen des höheren Gehaltes an Gerbstoffen bevorzugt verwendet. Es werden eine adstringierende, diuretische und tonisierende Wirkung auf die Gefässe genannt und für die einzelnen Zubereitungsformen die zu diesem Zeitpunkt üblichen Indikationen zusammengestellt (Tab. 2).

\section{Suche nach dem Wirkprinzip}

In der Folge versuchte man das Wirkprinzip des roten Weinlaubs zu charakterisieren. Neben den Gerbstoffen sah man auch die Anthocyane, die Ursache der Rotfärbung, als wirksam an. Eine pharmakologisch-klinische Studie zu einem Anthocyan-haltigen Fertigpräparat aus rotem Weinlaub (OpoVeinogene von Maison Biosedra) ist 1953 in Frankreich erschienen, in der die Wirksamkeit bei Krampfadern, vegetativer Dystonie im Zusammenhang mit der Menopause und lokalen Durchblutungsstörungen der oberen Extremitäten beschrieben und auf die weiter unten erklärte Vitamin-P-Wirkung zurückgeführt wird - mit Anthocyanen als dem gefässwirksamen Prinzip [64].

\section{Monographie}

Aufgrund der gut dokumentierten medizinischen Verwendung wurde rotes Weinlaub 1986 in Frankreich in die offizielle Liste der traditionell genutzten Heilpflanzen mit den Indikationen Kapillarbrüchigkeit, Veneninsuffizienz, Hämorrhoiden und Augenreizungen aufgenommen [1]. Eine Qualitätsmonographie für rotes Weinlaub findet sich seit 1996 unter der Bezeichnung «Vigne rouge» in der Pharmacopée française [52]. Aktuell sind in der französischen Pharmaliste VIDAL mehrere Produkte mit rotem Weinlaub meist als Tee oder Kapseln mit Drogenpulver und Extrakt, aber auch als Tropfen oder Augenbad aufgelistet [62].

Erste Hinweise auf die Anwendung von rotem Weinlaub ausserhalb Frankreichs finden sich 1957 in Italien. Auch dort hat man das Präparat von Biosedra bei Gefässerkrankungen in der Gynäkologie getestet und dabei eine gute Wirkung auf die Kapillarbrüchigkeit gefunden [8].

Bereits 1960 erschien in Deutschland eine klinische Studie zur Venenwirksamkeit für das Produkt Pedopur
Tab. 2. Indikationen für rotes Weinlaub (Frankreich, 1948) [16]

\begin{tabular}{|l|}
\hline Als Tee bei \\
- Durchfall \\
- Ruhr \\
- Bluterguss \\
- Harnverhalten \\
- Gicht \\
- Erbrechen \\
- Hautausschlag \\
- Krampfadern \\
Als Drogenpulver bei \\
- hartnäckigen Blutergüssen \\
- Uterusblutungen \\
- häufigem Nasenbluten \\
\hline
\end{tabular}

der Firma Rugard, Köln, das einen Fluidextrakt aus rotem Weinlaub mit einem DEV von 1:1 enthielt. Es war auf einen Inhaltsstoffgehalt von 600 gamma-Anthocyan pro $\mathrm{ml}(600 \mu \mathrm{g} / \mathrm{ml})$ eingestellt [23]. Später änderte man diese Einstellung auf 1,2 mg Bioflavonoide pro ml (Rote Liste 1965).

\section{"Vitamin P»}

Anthocyane und Flavonoide erkannte man als chemisch strukturverwandt und hielt sie beide für das Wirkprinzip, was vor dem Hintergrund der damaligen Diskussion um das Vitamin P zu sehen ist. Der Begriff «Vitamin P» wurde von dem ungarischen Nobelpreisträger Albert Szent-Györgyi geprägt im Zusammenhang mit der Wirksamkeit des Flavonoids Citrin bei Erkrankungen mit hämorrhagischen Erscheinungen [54]. Die getestete Substanz wurde Vitamin P (P für Permeabilität) genannt, da sie die Durchlässigkeit der kapillaren Blutgefässe normalisierte. Aus Tierversuchen hat man zunächst auf eine Mangelerscheinung geschlossen, musste aber den Vitaminstatus wieder fallen lassen, da sich die Flavonoide als nicht essenziell erwiesen [46]. Aber dieser Trugschluss hat die weitere Erforschung der Flavonoide und ihrer Wirkung auf die Gefässe angeregt.

Nachfolger des Produktes Pedopur in Deutschland ist ein Arzneimittel mit der Bezeichnung Antistax, das sich seit 1971 im Markt befindet. Die Kapseln und Tabletten enthalten 180 und $360 \mathrm{mg}$ Trockenextrakt aus roten Weinrebenblättern. 1969 hat die Firma Planta Subtil, Oldenburg, das Präparat als Arz- neimittel mit den Anwendungsgebieten Varizen, Phlebitis, Thrombophlebitis, Wadenkrämpfe und Ödeme in den Beinen registriert. Im Februar 1996 hat die Firma Boehringer Ingelheim das Produkt übernommen und seither in mehreren europäischen Ländern mit der Indikation Vorbeugung und Behandlung chronisch-venöser Insuffizienz (CVI), Krampfadern, Beinödeme, schwere Beine eingeführt.

\section{Pharmakologie}

Die Wirksamkeit des Extraktes aus rotem Weinlaub wird heute auf den Gehalt an den Flavonoiden Quercetin-3O- $\beta$-D-glucuronid, Isoquercitrin (Quercetin-3-0- $\beta$-D-glucosid) und Kaempferol-3-O- $\beta$-D-glucosid zurückgeführt. Neuere klinische Studien belegen die Wirksamkeit von rotem Weinlaubextrakt zur Ödemreduktion bei chronischvenöser Insuffizienz und zeigen eine Steigerung der Mikrozirkulation der Haut bei verbesserter Sauerstoffversorgung [28,30]. In einer Anwendungsbeobachtung [55] konnte eine Verringerung der subjektiven CVI-Symptome, wie müde, schwere Beine, Spannungsgefühl, Kribbeln und Schmerz in den Beinen, gezeigt werden. Bei pharmakologischen Untersuchungen des Extraktes und der genannten Flavonoide erkannte man als Wirkort das Endothel der Gefässe, wobei das Öffnen der Endothelialbarriere in den Venen und damit die Extravasation sowohl akut als auch prophylaktisch verhindert wird [48].

\section{Verwendung von Weinlaub als Lebensmittel}

Nicht unerwähnt bleiben soll die Verwendung von Weinblättern in der Lebensmittelbereitung, wofür in Frankreich der Begriff «application a l'art culinaire» (Anwendung in der kulinarischen Kunst) verwendet wird [36]. Der Verzehr der Blätter ist hauptsächlich in den Ländern des Nahen Ostens üblich. Bekannt ist auch bei uns die türkisch-griechische Spezialität Dolma oder Dolmades: Rouladen in Weinblatthülle, gefüllt mit gehacktem Hammelfleisch, Rebhühnern oder gewürztem Reis, frisch oder in Öl eingelegt [7]. Auf diese Art der Verwendung in Ägypten 
wird bereits 1740 hingewiesen [42]. Das Einschlagen von Käse in Weinblätter ist z.B. bei den Spezialitäten Picón Bayes-Tresviso (Spanien) oder Epoisses de Bourgogne (Frankreich) üblich und als Qualitätszeichen bekannt. Mit diesen Spezialitäten haben Weinblätter auch in unsere Küchen Einzug gehalten. Als Beleg dafür kann man anführen, dass die Warenaufstellung für Sammelzollverfahren «Weinblätter, zur menschlichen Ernährung» nennt [38]. Franken als Weinland darf bei so vielen Spezialitäten nicht zurückstehen und kreierte deshalb die Bratwurst im Weinlaub (dpa, SZ 13.8.2005).

\section{Danksagung}

Den genannten Bibliotheken sei für die Erlaubnis zur Reproduktion der Abbildung gedankt. Hinweise auf Literaturstellen und Hilfe bei der Interpretation haben dankenswerterweise gegeben: Frau Dr. R. Achenbach, UB Regensburg, Frau B. Mund, UB Göttingen, Frau S. Muth, Bibliothek Forschungsanstalt Geisenheim, Herr Dr. S. Grotz, Institut für Philosophie, Universität Regensburg und Herr Dr. F. Schumann, Gesellschaft für Geschichte des Weines.

\section{Trilogie}

Die vorliegende Übersicht zum Roten Weinlaub ist im Rahmen einer Trilogie erschienen: Über die pharmazeutische Qualität und klinische Wirksamkeit informiert der Beitrag E. Schneider: Rotes Weinlaub - eine venenwirksame Arzneidroge. Dtsch Apoth Ztg 2007; 147(39): 3380-3386. Die Phytochemie ist zusammengefasst bei: E. Schneider, $\mathrm{H}$ von der Heydt, A. Espereste: Evaluation of polyphenol composition in red leaves from different varieties of Vitis vinifera L. ssp. vinifera. Planta Med 2008;74:565-572

\section{Literatur}

1 Agence du Médicament: Médicaments à Base de Plantes. Les Cahiers de l'Agence 3, Saint-Denis, Paris, 1998.

2 Bauhin C: PINAX - Theatri botanici. Basel; 1671

3 Baumann $\mathrm{H}$ : Pflanzenbilder auf griechischen Münzen. München: Hirmer; 2000.

4 Bedford-Stundenbuch. British Library London. [Reprint] mit wissenschaftlichem Kommentar von Eberhard König. Faksimile Verlag Luzern: 2005.

5 Berendes J: Dioscurides Materia medica. Stuttgart;1902.

6 Berg OC: Charakteristik der für die Arzneikunde und Technik wichtigsten Pflanzen-Gattungen in Illustrationen. Berlin: Rudolph Gaertner; 1845.

7 Bergner KG, Lemperle E: Weinkompendium, Botanik - Sorten - Anbau - Bereitung. Stuttgart: Hirzel: 1993

8 Bertone C: L'opo-fitoterapia nelle vasculopatie incidenti in campo ostetrico e ginecologico. Minerva Ginecologica 1957:9:879-881.

9 Bock H: Kreütterbuch. Straßburg: Rihel; 1577. [Reprint] München: Kölbl: 1964

10 Bombardelli E, Morazzoni P: Vitis vinifera L. Fitoterapia 1995:66:291-317.

11 Brun JP, Laubenheimer F: La vin et al vigne en Gaule. L'Archéologue 2003:63:4-14

12 Chomel JB: Supplément a l'abrégé de I'histoire des plantes usuelles. Paris; 1730 [2.ed. 1804]

13 Dodoens R: Cruydt-boeck Remberti Dodonaei. Antwerpen; 1644. [Reprint] Delft: Rijperman; 1980.
14 Dressendörfer W: Blüten, Kräuter und Essenzen. Heilkunst alter Kräuterbücher. Ostfildern: Thorbecke; 2003.

15 Fischer H: Mittelalterliche Pflanzenkunde. München: Verlag der Münchner Drucke; 1929.

16 Fournier $P$ : Le livre des plantes médicinales et vénéneuses de France. 1.500 espèces par le texte et par l'image d'après l'ensemble de nos connaissances actuelles. Paris: Lechevalier; 1948.

17 Fuchs L: De historia stirpium commentarii insignes maximis impensis et vigiliis elaborati adjectis earundem vivis plusquam quingentis imaginibus, numquam antea ad naturae imitationem artificiosus effictis et expressis. Basel; 1542. Folio. UB Regensburg. Sig. 241/W758669, Rara der RBG.

18 Galet P: Cépages et vignobles de France. Tome 2. Montpellier: Dehan; 1990.

19 Galet P: Dictionnaire encyclopédique des cépages. Paris: Hachette; 2000

20 Germer R: Untersuchung über Arzneimittelpflanzen im Alten Ägypten. [Dissertation] Hamburg: Universität; 1979

21 Godlewsi G, Blanque G: La vigne rouge en thérapeutique toni-veneuse. Gazette médicale de France, Sept. 1943.

22 Goetz P: Vigne rouge: de l'ethnopharmacologie à I'utilisation en phytothérapie. Phytothérapie 2003:1:42-44.

23 Güthenke K: Wesen und Behandlung der Bindegewebsschwäche, insbesondere der Veneninsuffizienz mit Weinblattextrakt. Dtsch Med J 1960; 11:370-373.

24 Helmstädter A: Pflanzenforschung als Passion. Pharm Ztg 2007:152:28.

25 Hoppe HA: Drogenkunde, Bd.1, Angiospermen. Berlin: de Gruyter; 1975

26 Horst J: Opusculum de vite vinifera ejusque partibus. Marpurgi: Chemlin; 1630

27 Jessen K: Albertus Magnus De vegetabilibus libri VII, historiae naturalis pars XVIII. Berlin: Reimer: 1867, Buch VI § 236-256.

28 Kalus U, Koscielny J, Grigorov A, et al.: Improvement of cutaneous microcirculation and oxygen supply in patients with chronic venous insufficiency by orally administered extract of red vine leaves AS 195: a randomised double-blind placebo-controlled, crossover study. Drugs R D 2004;5:63-71.

29 Keller-Meister [anonym]: Der Zu allerley guten Geträncken treuhertzig-anweisende wohlerfahrne und Curiose Keller-Meister. [Th. 1. 2.] Nürnberg: Lochner: 1705-1708.

30 Kiesewetter $\mathrm{H}$, Koscielny J, Kalus $U$, et al.: Efficacy of orally administered extract of red vine leaf AS 195 in chronic venous insufficiency (stages I-II). Arzneimittelforschung 2000;50:109-117.

31 Kommission D: Monographie Vitis vinifera. Bundesanzeiger Nr. 104 a vom 7.6.1990.

32 König $P$, Weiland J: Arzneimittelprüfung Wein: Vitis vinifera; zwei homöopathische Arzneimittelstudien. Rösrath: Fagus-Verlag Wichmann; 2003.

33 König R: Plinius Naturkunde, lateinisch - deutsch. Bd.24. Darmstadt: Wiss. Buchges.; 1993

34 Kühn CG (Hrsg.): Claudii Galeni opera omnia. Hildesheim: Olms; 1965

35 Lambertus von St Omer: Liber floridus, ca. 1120. Wolfenbüttel: Herzog August Bibliothek, [Digitalisierte Handschriften] Codices Gudiani sive graeci sive latini, Cod. Guelf. 1 Gud. lat. Blatt 158 im Internet aufgerufen unter http://www hab de/ bibliothek/wdb/ mssdigital.htm

36 Leclerc H: La feuille de Vigne. Revue de Phytothérapie (Paris) 1942, (5):5-10.

37 Leclerc $\mathrm{H}$ : Précis de Phytothérapie - Essais de Thérapeutique par les Plantes Françaises. 4.ed. Paris: Masson; 1954

38 Liebster G: Warenkunde Obst und Gemüse. Bd.2 Gemüse. Düsseldorf: Morion; 1990.

39 Lieutaghi P: L'Herbe qui renouvelle. Un aspect de la médecine traditionnelle en Haute-Provence. Paris: Editions de la Maison des Sciences de I'Homme; 1986.

40 Linnaeus C: Species Plantarum - A Facsimile of the First Edition 1753. London: Ray Society: 1959

41 Lonicerus A: Kreuterbuch. Frankfurt: Uffenbach: 1679. [Reprint] München: Kölbl: 1962.

42 Maillet B: Description de l'Egypte / composée sur les mémoires de Monsieur de Maillet par Le Mascrier. Vol 1. La Haye: Beauregard; 1740.

43 Mania D: Die ersten Menschen in Europa. Ar- chäologie in Deutschland, Sonderheft, Stuttgart: Theiss: 1998

44 Martinus Opifex: Tacuinum sanitatis Handschrift Regensburg um 1445. In: Tomás de Cantimpré: (Codex granatensis). De natura rerum, de Tomás de Cantimpré. De avibus nobilibus. Tacuinum sanitatis, de Ibn-Butlán. Granada: Biblioteca Universitaria, Sign.: C 67.

45 von Megenberg C: Buch von der Natur. Augsburg: Johann Schönsperger; 1499. Frontispiz Von den bäumen. Wolfenbüttel: Herzog August Bibliothek [Digitalisierte Handschriften]. http://diglib hab de/wdb php?dir=inkunabeln/45-1-phys-2f

46 Moll T: Zur Frage des Vitamin P. J Mol Med 1937; 16:1653.

47 Müller-Jahncke WD: Ordnung durch «Signatur». Analogiedenken im Arzneischatz des 16. und 17 Jahrhunderts. Dtsch Apoth Ztg 1984;124:2184-89.

48 Nees S, Weiss DR, Reichenbach-Klinke $E$, et al.: Protective effects of flavonoids contained in the red vine leaf on venular endothelium against the attack of activated blood components in vitro. Arzneimittelforschung 2003; 53:330-341.

49 Németh $M$ : Ampelográfiai album; alany- direkt termö és csemegeszölö-fajták; függelék. Budapest: Mezögazdasági Kiadó; 1975.

50 Ortus sanitatis. [Nachdr. der Ausg. Venedig 1511] Würzburg: Edition Popp; 1978, Bild + Text: CCCCXCIIII.

51 Pabst G: Köhler's Medizinal-Pflanzen in naturgetreuen Abbildungen mit kurz erläuterndem Texte. Atlas. Gera: Verlag Fr. Eugen Köhler; 1887.

52 Pharmacopée française, 10ème édition. Agence Française de Sécurité Sanitaire des Produits de Santé. Monograph Vigne rouge, 1996.

53 Riethe P: Hildegardis Bingensis Naturkunde, das Buch von dem inneren Wesen der verschiedenen Naturen in der Schöpfung. Salzburg: Müller; 1959.

54 Rusznyák S, Szent-Györgyi A: Vitamin P. Flavonols s vitamins. Nature 1936;138: 27

55 Schäfer E, Peil H, Ambrosetti L, Petrini O: Oedema protective properties of the red vine leaf extract AS 195 (Folia vitis viniferae) in the treatment of chronic venous insufficiency. A 6-week observational clinical trial. Arzneimittelforsch 2003;53:243-46.

56 Schipperges H: Hildegardis Bingensis Heilkunde, das Buch von dem Grund und Wesen und der Heilung der Krankheiten. Salzburg: Müller; 1957.

57 Schröder JD: Johann Schröders/Pharmacopoeia Universalis,/Das ist:/Allgemeiner MedicinischChimischer/Artzney-Schatz/Th.1-3. Nürnberg; 1746-1748 (Bibliothek des Deutschen Museums, München; Sign. 1929 B 906).

58 Schwabe W: Homöopathisches Arzneibuch. 2. Aufl. Leipzig, 1934.

59 Tabernaemontanus JT: Neu vollkommen KräuterBuch 1731. [Reprint] München: Kölbl: 1975.

60 Tournefort JP: Josephi Pitton Tournefort, Institutiones rei herbariae. Tomus I + III / ed. tertia appendicibus aucta ab Antonio de Jussieu, l'éd. de, Parisiis: Typographia regia, 1719. Im Internet aufgerufen unter www.gallica.fr

61 Viala P Vermorel V: Ampélographie - Traité Général de Viticulture, Vol. 1-7. Paris: Ed. Masson et Cie; 1905-1910.

62 VIDAL Liste des Médicaments, 2005. www.vidal.fr

63 Wonnecke von Kaub J: Hortus sanitatis Mainz: Peter Schöffer, 1485. [Faks.-Ausg.] München: Verlag der Münchner Drucke: 1924, Bild + Text: CCCCXVI vitis - wyn reben.

64 Zicavo B: Les anthocyanes, facteurs d'utilisation des sucres et leur effets circulatoires. Phlébologie 1953;6:51-58.

65 Zohary D, Spiegel-Roy P:Beginnings of fruit growing in the Old World. Olive, grape, date, and fig emerge as important Bronze Age additions to grain agriculture in the Near East. Science 1975 187:321-323.

\section{Anschrift des Autors}

Dr. Ernst Schneider

PhytoConsulting

Seeblick 11, D-84163 Marklkofen-Freinberg

schneider.e@phyto-consulting.de

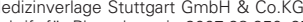

